\title{
Complications of contralateral C-7 transfer through the modified prespinal route for repairing brachial plexus root avulsion injury: a retrospective study of $\mathbf{4 2 5}$ patients
}

\author{
Wenjun Li, MD, PhD, ${ }^{1}$ Shufeng Wang, MD, PhD, Jianyong Zhao, MD, ${ }^{1}$ \\ M. Fazlur Rahman, MD, ${ }^{3}$ Yucheng Li, MD, ${ }^{1}$ Pengcheng Li, MD,, and Yunhao Xue, MD1 \\ ${ }^{1}$ Department of Hand Surgery, Beijing Jishuitan Hospital, Peking University, Beijing; ${ }^{2}$ Department of Hand Surgery, Cangzhou \\ Hospital of Integrated TCM and WM Hospital, Hebei Medical University, Cangzhou, China; and ${ }^{3}$ Department of Hand Surgery, \\ Aga Khan University Hospital, Karachi, Pakistan
}

\begin{abstract}
OBJECT In this report, the authors review complications related to the modified prespinal route in contralateral C-7 transfer for repairing brachial plexus nerve root avulsion injury and suggest a prevention strategy.

METHODS A retrospective, nonselected amalgamation of every case of modified contralateral C-7 transfer through the prespinal route was undertaken. The study population comprised 425 patients treated between February 2002 and August 2009. The patients were managed according to a standardized protocol by one senior professor. The surgical complications were grouped into one of the following categories: those associated with tunnel making through the prespinal route, those related to the dissection and transection of the contralateral C-7 nerve root, and those that occurred in the postoperative period.
\end{abstract}

RESULTS The study population included 379 male and 46 female patients whose average age was 21 years (range 3 months to 56 years). A total of 401 patients were diagnosed with traumatic brachial plexus injury, the leading cause of which was motor vehicle accident, and 24 patients were diagnosed with obstetrical brachial plexus palsy. The contralateral C-7 nerve root was cut at the proximal side of the division portion of the middle trunk in 15 cases and sectioned at the distal end of the anterior and posterior divisions in 410 cases. The overall incidence of complications was $5.4 \%$ (23 of 425). Complications associated with making a prespinal tunnel occurred in 12 cases, including severe bleeding due to vertebral artery injury during the procedure in 2 cases $(0.47 \%)$, temporary recurrent laryngeal nerve palsy in 5 cases $(1.18 \%)$, pain and numbness in the donor upper extremity during swallowing in 4 cases $(0.94 \%)$, and dyspnea caused by thrombosis of the brainstem 42 hours postoperatively in 1 case $(0.24 \%)$; this last patient died 38 days after the operation. Complications related to exploration and transection of the contralateral C-7 nerve root occurred in 11 cases, including deficiency in extensor strength of the fingers and thumb in 4 cases $(0.94 \%)$ due to injury to the posterior division of the lower trunk, unbearable pain on the donor upper extremity in 3 cases $(0.71 \%)$, Horner's syndrome in 2 children $(0.47 \%)$ who suffered birth palsy, a section of C-6 nerve root mistaken as C-7 in I case $(0.24 \%)$, and atrophy of the sternocostal part of the pectoralis major in 1 case $(0.24 \%)$.

CONCLUSIONS The most serious complications of using the modified prespinal route in contralateral C-7 transfer were vertebral artery laceration and injury to the posterior division of the lower trunk. The prevention of such complications is necessary to popularize this surgical procedure and attain good long-term clinical results.

http://thejns.org/doi/abs/10.3171/2014.10.JNS131574

KEY WORDS brachial plexus repair; contralateral C-7 nerve root; vertebral body; complication; root avulsion; peripheral nerve

ABBREVIATION CC-7 = contralateral C-7.

SUBMITTED July 31, 2013. ACCEPTED October 21, 2014.

INCLUDE WHEN CITING Published online December 12, 2014; DOI: 10.3171/2014.10.JNS131574.

DISCLOSURE The authors report no conflict of interest concerning the materials or methods used in this study or the findings specified in this paper. The following grants funded this study: Grant No. 2007-3066 from the Beijing Medicine Research and Development Fund and Grant No. 2012-012 from the open projects of the Key Laboratory of Hand Reconstruction of the Ministry of Health and the Shanghai Key Laboratory of Peripheral Nerve and Microsurgery. 
$\mathrm{P}$ REGANGLIONIC injury (root avulsions) to all 5 roots of the brachial plexus is, unfortunately, a common presentation. Reconstruction to reestablish function, however, remains a daunting challenge. Surgery inevitably entails the transfer of nerves from outside the brachial plexus. Use of the C-7 root from the opposite, uninjured brachial plexus, was first proposed by Gu et al. in $1986 . .^{7}$ Over the past 2 decades, several studies verified the safety and effectiveness in the clinical application of this technique..$^{2-7}$ The chief pitfall has always been the long delay in manifestation of results caused by the distance that the growing axons have to travel along vascularized or nonvascularized nerve grafts placed in subcutaneous tunnels across the neck and chest. McGuiness and Kay ${ }^{10}$ first addressed this issue when they reported on a patient with obstetrical palsy in whom they used nerve grafts passed along the prespinal retropharyngeal route to bridge the posterior half of the opposite $\mathrm{C}-7$ to the median nerve. They mentioned the advantages of easier dissection, shorter nerve grafts, and a more protected placement of the grafts. However, they missed mention of an earlier report by Wang (the senior author of this paper) and colleagues ${ }^{11}$ in February 2002 describing the transfer of the entire C-7 root via the prevertebral route deep to the scalenus anterior and the longus colli muscles. In 2008, Xu et al. ${ }^{13}$ modified the McGuiness-Kay technique by transecting the scalenus anterior muscle on both sides. This was done to shorten the length of the nerve graft and to produce a smoother passage in the retroesophageal space. The prespinal passage described by Wang et al. (Fig. 1)

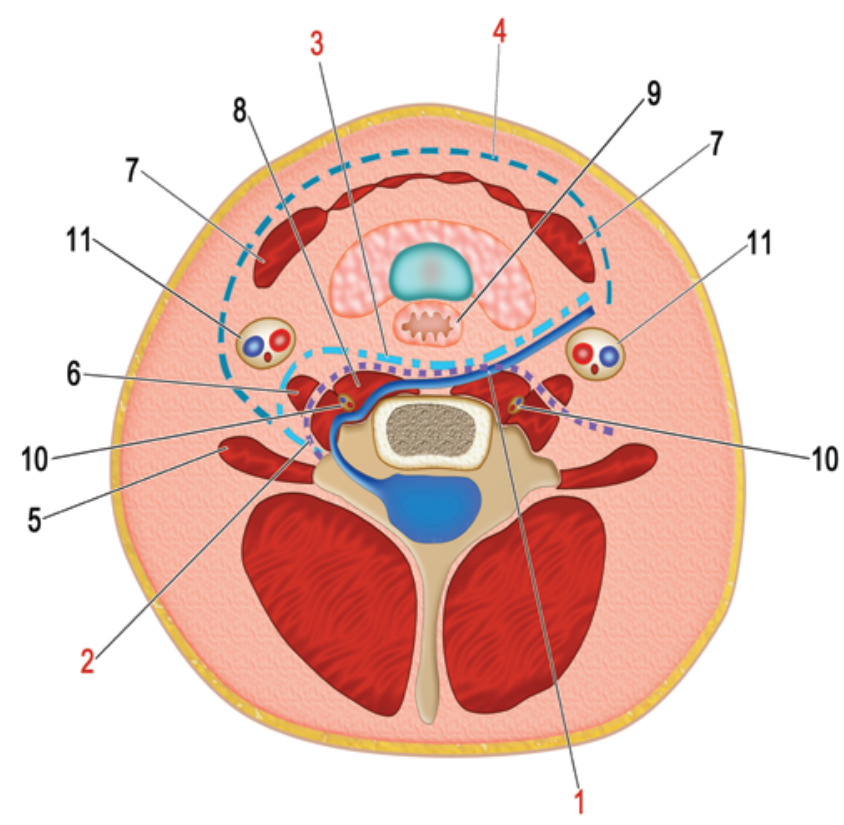

FIG. 1. Schematic of the $\mathrm{cC}-7$ nerve root transfer via the prespinal route: 1 = our modified prespinal route; 2 = prespinal route designed by Xu et al.; 3 = prespinal route designed by McGuiness and Kay; 4 = cervical subcutaneous tunnel of traditional cC-7 nerve transfer; $5=$ scalenus median muscle; 6 = scalenus anterior muscle; 7 = sternocleidomastoid muscle; 8 = longus colli; 9 = esophagus; 10 = vertebral artery and vein; $11=$ carotid sheath. Copyright Xiaoping Li. Published with permission. Figure is available in color online only. is the shortest route, and it allows for transfer of the C-7 root to a position in which direct coaptation to the lower trunk $^{12}$ or the C-5 and C-6 nerve roots ${ }^{11}$ on the injured side is feasible. However, this method is not devoid of complications. We studied these complications in a series of 425 patients who underwent systematic transfer of the opposite C-7 nerve root by the prespinal route for root avulsion injuries of the brachial plexus.

\section{Methods}

This study comprises 425 patients who underwent surgery between February 2002 and August 2009. There were 46 female and 379 male patients who ranged in age from 3 months to 56 years (mean 21 years). Each patient was followed up for a minimum period of 12 weeks after the operation.

All patients had suffered a brachial plexus injury that did not show signs of spontaneous recovery within 3-6 months from the accident (or birth). The indications for opposite C-7 nerve transfer via the prespinal route included total or near-total brachial plexus avulsion injuries with or without spinal accessory or phrenic nerve lesions. Each procedure was performed according to the steps outlined in our previous report. ${ }^{11}$ The chief operating surgeon was the senior author (S.W.), and 2 doctors from the Department of Hand Surgery assisted him.

Details of the examinations, operative notes, and postoperative course in the hospital were meticulously recorded for each case. All the hospital charts were available for review. Each diagnosis was based on detailed clinical evaluation, electrophysiological study, and CT myelography findings and confirmed by surgical exploration. Complete attending physician records of all patients were also available for examination. All records were reviewed to determine demographic data, primary diagnosis, clinical results, and intra- and postoperative complications. With such a massive number of cases in a retrospective study, follow-up is always hard work, but for the convenience of data collection, since the first case of contralateral C-7 (cC-7) nerve transfer, we have written a note for every patient, including information on the intraoperative findings, the surgical procedure, and intraoperative complications immediately after surgery. From the 1st day after surgery to discharge of each patient, our group members add new information in the notes regarding new symptoms such as hoarseness, Horner's sign, pain, decreased muscle strength, and so on. For patients who experienced complications, the follow-up time was more than 3 years. For those without complications, the follow-up was at least 12 weeks. All patients data were collected by a doctor who assisted in surgery, and eventually, the patient's information was summarized and stored with some images to contribute to the follow-up.

Traumatic brachial plexus injury was diagnosed in 401 cases. Of these, 112 cases were treated with a sural nerve graft for bridging the upper trunk, and 24 cases were treated using a nerve graft for bridging between $\mathrm{cC}-7$ with the median nerve, medial cord, lower trunk, or C-8 nerve root. In 4 patients, the $\mathrm{cC}-7$ nerve was coapted directly to the C5-6 nerve root on the injured side. A total of 261 cases 
were treated with direct coaptation of $\mathrm{cC}-7$ nerve and the lower trunk or medial cord.

Obstetrical palsy was diagnosed in the 24 patients, in whom nerve grafts were placed. The $\mathrm{cC}-7$ nerve was bridged to the upper trunk or its anterior and posterior divisions in 12 cases; the medial cord, lower trunk, or C-8 nerve root in 11 cases; or the posterior division of the affected middle and lower trunk and the C-7 nerve root in 1 case.

The cC-7 nerve transfer was always performed via the prespinal route. In 15 cases, the C-7 nerve was divided at the proximal part of the divisions. In the remaining 410 patients, the it was dissected as distally as possible along its divisions before sectioning it.

Each complication of $\mathrm{cC}-7$ nerve transfer through the prespinal route was categorized into 1 of 3 groups (Table 1): complications resulting from preparation of the prespinal passage, complications resulting from sectioning of the cC-7 nerve, and general complications.

The complications related to the prespinal passage included hemorrhage, dyspnea, perforation of the esophagus, hoarseness, and pain and numbness in the healthy arm when the patient was swallowing. Accidental injury to the vertebral artery, the common carotid artery, or the internal jugular vein resulted in severe hemorrhage. Dyspnea occurred as a result of hematoma-related compression. Accidental injury to the recurrent laryngeal nerve was responsible for the hoarseness. Pain and numbness in the healthy arm was caused by compression of the nerve by esophagus peristalsis.

Complications of cC-7 transection occurred during and after surgery. Intraoperative complications included unintentional injury to other nerve roots or divisions that were identified immediately. Postoperative complications included any weakness in flexion or extension of the elbow, fingers, and thumb. Each patient was examined in detail after the operation, and records of such instances and their duration were maintained. Apart from these intraoperative complications, other incidents such as infection, lymphedema, or lymph leakage were also reviewed.

\section{Results}

All 425 patients were followed up, and for those 23 patients in whom complications occurred, follow-up was continuous for more than 3 years. For those patients in whom no complications occurred, the follow-up time was at least 12 weeks.

\section{Complications Associated With Making the Prespinal Tunnel}

Vessel Injury

In 2 cases $(0.47 \%)$, the vertebral artery was injured, and blood loss amounted to $600 \mathrm{ml}$ in one patient and $1000 \mathrm{ml}$ in the other. In 1 case, the artery was reconstructed using a vein graft. After the operation, this patient complained of mild headache and dizziness that improved spontaneously within 4 days. This patient did not suffer any discomfort in the movement of his limbs, and there were no other complaints. In the other case, the vertebral artery was ligated and divided, producing no apparent deficit. There was no incident involving injury to the common carotid artery or the internal jugular vein. We did not note serious hematoma in any patient.

\section{Hoarseness}

Five patients (1.18\%) experienced postoperative hoarseness after the operation, which resolved within 7 days in 4 patients and within 4 weeks in 1 patient.

\section{Upper-Limb Pain on the Noninjured Side While Swallowing}

Four patients $(0.94 \%)$ experienced numbness and pain in the upper limb contralateral to the injury side while swallowing. The symptoms were mild in 2 cases, disappearing after 4 postoperative days in one patient and 7 postoperative days in the other patient. In a third patient with numbness and pain, the symptoms diminished beginning on the 10th postoperative day and resolved after 2 weeks. In the fourth patient, when the symptoms did not diminish, an exploration was performed, and the anastomosis site between $\mathrm{cC}-7$ nerve and the bridging nerve was found to be ruptured. A sural nerve graft was harvested, and the defect was bridged using the graft. The patient's symptoms disappeared after this operation.

\section{Dyspnea}

One patient $(0.24 \%)$, treated with $\mathrm{cC}-7$ nerve root transfer through the prevertebral route to repair the upper trunk, suffered dyspnea 42 hours after surgery. Clinical examination revealed a partial palsy of the contralateral upper limb and weakness in the lower limbs and in the

TABLE 1. Complication categories

\begin{tabular}{|c|c|c|c|}
\hline \multirow[b]{2}{*}{ Tunnel Making } & \multicolumn{2}{|c|}{ cC-7 Nerve Dissection \& Transection } & \multirow[b]{2}{*}{ General } \\
\hline & Intraop & Postop & \\
\hline Hemorrhage & $\begin{array}{l}\text { Injury to other nerve roots } \\
\text { or divisions }\end{array}$ & Decreased elbow extension & Infection \\
\hline Dyspnea & & Decreased wrist extension & Lymphedema \\
\hline Esophagostoma & & Decreased finger extension & Lymph leakage \\
\hline Hoarseness & & Decreased finger flexion & \\
\hline \multirow[t]{3}{*}{ Numbness \& pain in healthy arm on swallowing } & & Pain in healthy upper extremity & \\
\hline & & Horner's syndrome & \\
\hline & & $\begin{array}{l}\text { Decreased pectoralis major } \\
\text { muscle strength }\end{array}$ & \\
\hline
\end{tabular}


muscles of the tongue. The reflexes were impaired in both lower limbs. Tracheal intubation was done, and the patient was transferred to the intensive care unit. The patient, however, died as a result of respiratory and cardiac failure.

We did not note any case of accidental perforation of the esophagus (Table 2).

\section{Complications Associated With cC-7 Nerve Root Dissection and Transection}

Incorrect Identification of the $\mathrm{CC}-7$ Nerve

In 1 case $(0.24 \%)$, after the C-7 nerve root was thought to be cut, we found another nerve root, which was the real cC-7 root. We dissected it carefully and found that there was an anatomical variation. The C-6 nerve root was horizontally divided into 2 rami just after it came out of the intervertebral foramen. The superior ramus joined the upper trunk with the anterior division of the C-5 nerve root. The inferior ramus divided into 2 branches, which converged into the anterior and posterior divisions of the superior trunk. This inferior ramus was mistaken to be the real C-7 nerve root and was cut. When the actual C-7 root was found, we decided to stop the operation. After the patient had woken up, we evaluated the muscle strength of the biceps brachii, which was found to be Grade 4 . The patient was anesthetized again, and the actual cC-7 nerve root was cut and transferred with the wrong-cut C- 6 inferior ramus divided earlier (Figs. 2 and 3). After the operation, the motor function of the patient's healthy upper arm was not apparently affected. There patient's experienced thumb, index finger, and middle finger numbness.

\section{Transection of the Posterior Division of the Lower Trunk}

In 1 case $(0.24 \%)$, the posterior division of the lower trunk was severed when we cut the posterior division of the $\mathrm{cC}-7$ root. We realized our mistake, but we thought at that time that it was not important for finger and thumb extension function, so we did not repair it. However, the patient did not recover finger and thumb extension strength.

\section{Decreased Elbow, Wrist, and Finger Extension Strength}

This complication was noted in 4 cases $(0.94 \%)$, which included the case of accidental injury to the posterior division of the lower trunk (mentioned earlier). In this patient, elbow and wrist extension recovered, but finger and thumb extension never returned (Fig. 4). In the other 3 cases, the average muscle strength of finger and thumb extensions was Grade 0, wrist extension was Grade 4-5, and

TABLE 2. Complications with tunnel making and outcomes

\begin{tabular}{lcc}
\hline \multicolumn{1}{c}{ Cause } & No. of Patients & Outcome \\
\hline $\begin{array}{c}\text { Hemorrhage after verte- } \\
\text { bral artery injury }\end{array}$ & 2 & Recovered \\
\hline $\begin{array}{l}\text { Dyspnea after thrombosis } \\
\text { of the brainstem }\end{array}$ & 1 & $\begin{array}{c}\text { Patient died of respiratory } \\
\text { \& cardiac failure }\end{array}$ \\
\hline Esophagostoma & 0 & All recovered \\
\hline Hoarseness & 5 & All recovered \\
\hline $\begin{array}{l}\text { Numbness \& pain on } \\
\text { healthy upper arm dur- } \\
\text { ing swallowing }\end{array}$ & 4 & \\
\hline
\end{tabular}

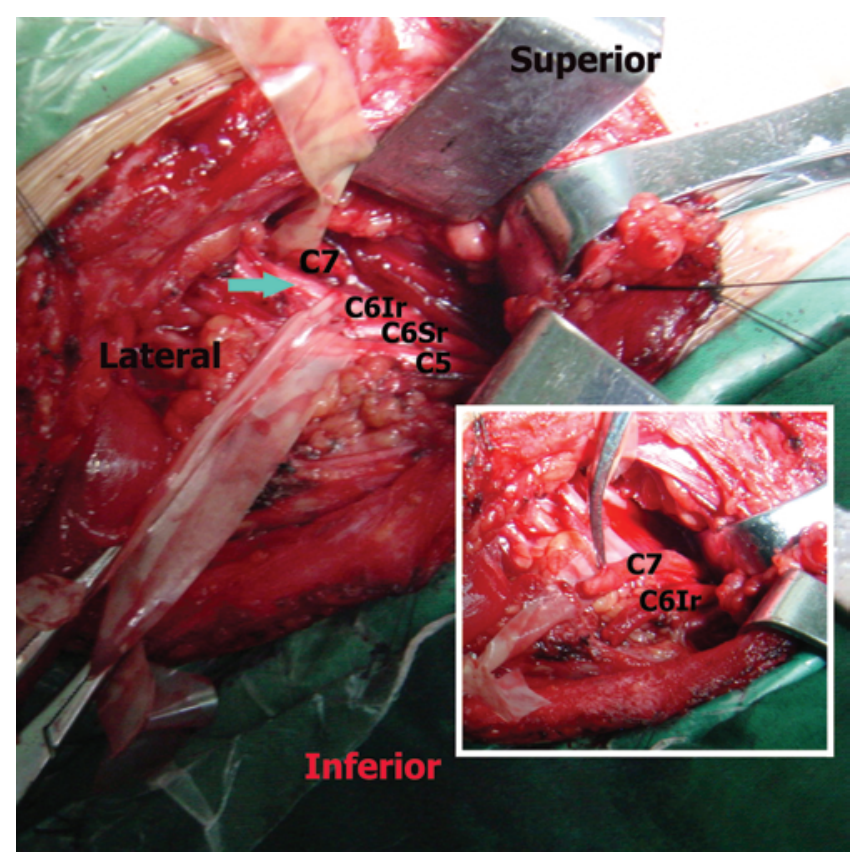

FIG. 2. The contralateral variant $\mathrm{C}-6$ nerve root was cut by mistake: $\mathrm{C} 6 \mathrm{Ir}$ $=$ inferior ramus of the variant $\mathrm{C}-6 ; \mathrm{C} 6 \mathrm{Sr}=$ superior ramus of the variant C-6. Arrow shows the cut point of the variation of C-6. Superior, inferior, and lateral are the orientations. Figure is available in color online only.

elbow extension was Grade 2-3 after surgery. These 3 patients recovered their muscle power up to Grade 3-4 in 4 months. However, 1 patient $(0.24 \%)$ had atrophy of the sternocostal part of the pectoralis major muscle (Fig. 5).

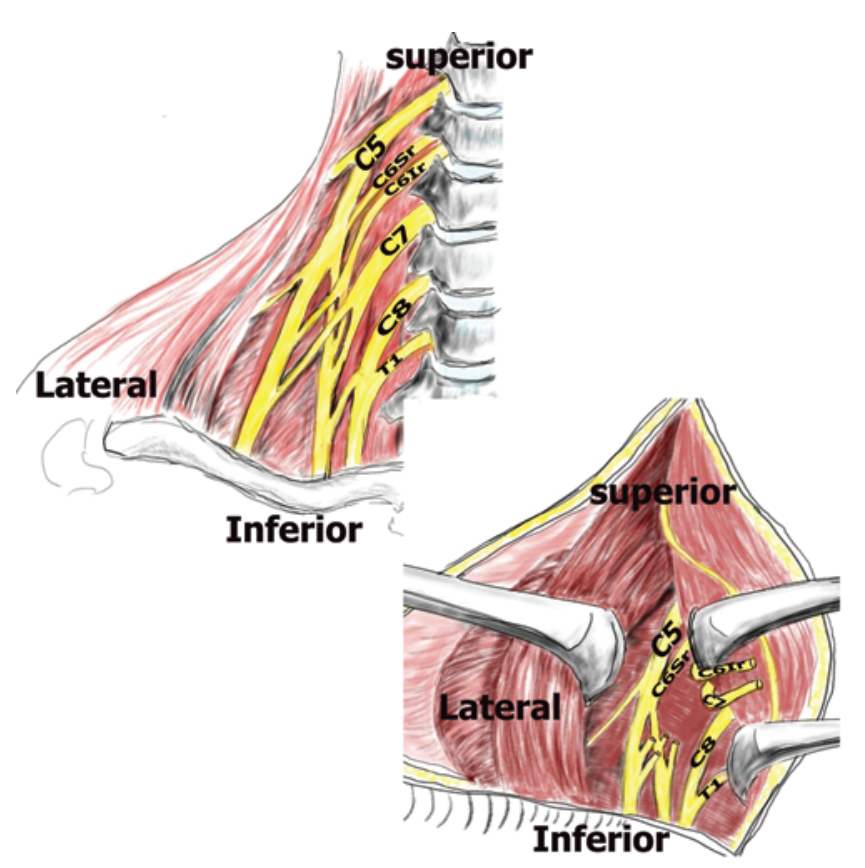

FIG. 3. Schematics of the contralateral variant C-6 nerve root, which was cut by mistake. $\mathrm{C} 6 \mathrm{Ir}=$ inferior ramus of the variant $\mathrm{C}-6 ; \mathrm{C} 6 \mathrm{Sr}=$ superior ramus of the variant $\mathrm{C}-6$. Superior, inferior, and lateral are the orientations. Copyright Xiaoping Li. Published with permission. Figure is available in color online only. 

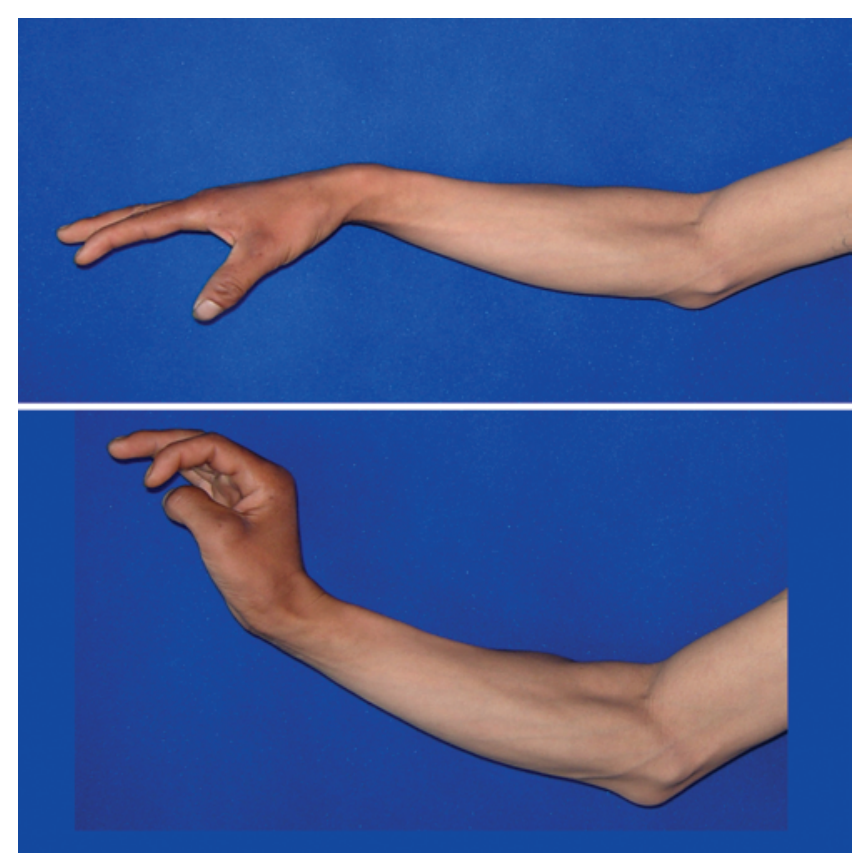

FIG. 4. Five years after the posterior division of the lower trunk was cut when taking the C-7 nerve root, normal function of wrist extension but dysfunction of thumb and finger extension remain. Figure is available in color online only.

\section{Severe Pain in the Contralateral Upper Extremity}

Three patients $(0.71 \%)$ complained of severe pain in the shoulder, back, and lateral upper arm on the healthy side within 3-5 days after surgery. In 2 of these patients, pain relief occurred 3 weeks later without any medication. The

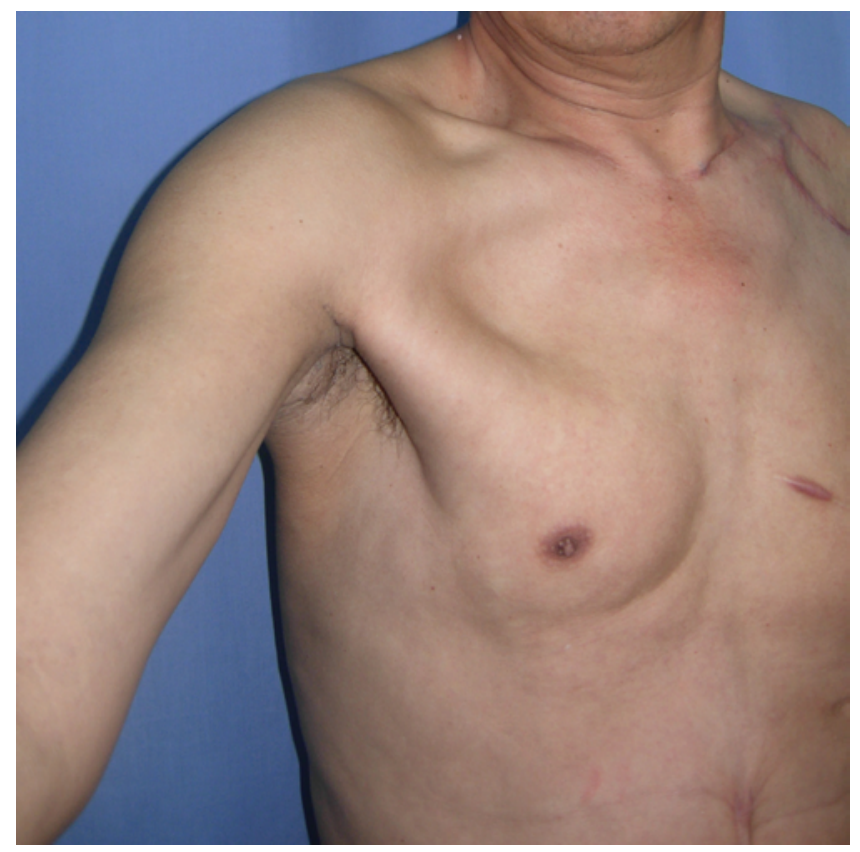

FIG. 5. One year after the cC-7 nerve section. Atrophy of the sternocostal part of the pectoralis major on the healthy (right) side. Figure is available in color online only. third patient needed oral medications and experienced pain relief 2 months after surgery.

\section{Horner's Syndrome}

Two 4-month-old patients $(0.47 \%)$, who were diagnosed with obstetrical brachial plexus palsy, got Horner's syndrome on the healthy side. One patient recovered fully within 3 months after surgery. The other patient had no ptosis or miosis 3 months after surgery but still had anhidrosis (Table 3).

\section{Other Complications}

We did not note any cases of infection, lymphedema, or lymphatic leakage.

\section{Discussion}

Complete brachial plexus avulsion injuries are devastating injuries with no easy solutions. In the absence of available nerve root stumps on the injured side, harvesting nerves from outside the brachial plexus or from the contralateral noninjured upper limb is the only option. There is no doubt that better function can be achieved if the transfer is performed closer to the target muscle. ${ }^{3,5,7}$ The cC-7 nerve transfer was introduced by $\mathrm{Gu}$ et al. ${ }^{7}$ in 1986. However, it has not gained popularity in the Western world because of fears that it will lead to dysfunction of the healthy donor limb. Passing the cC-7 nerve root in the retropharyngeal area can also lead to injury to the surrounding structures and result in pressure effects caused by compression of the nerve in that area. In such cases, the risk of complications may well seem very high. In this study, we examined the complications of this nerve transfer technique in a large number of patients.

We categorized each complication into 1 of 3 groups.

\section{Complications Arising From the Creation of the Retroesophageal Passage}

We evaluated our patients for injuries to the structures at risk (e.g., the esophagus and vertebral artery). Such complications occurred in 12 patients. There was no esophageal injury in any case, and although hoarseness did develop in 5 cases, it resolved in all of the patients. Pain and paresthesia in the upper limb on the noninjured side were noted in 4 patients. These symptoms improved spontaneously in 3 patients. In the fourth patient, the repair site was explored; it had given way, and revision was necessary.

The vertebral artery was injured in 2 cases $(0.47 \%)$. In both instances, the patients did not suffer any long-term effects after surgery. This injury can be deadly and difficult to manage. It is one of the most serious complications of nerve transfer via the prespinal route. If the artery is injured, the first step should be to control the bleeding, which can be achieved by direct pressure toward the vertebral body or laterally. The site of injury should be carefully located and dealt with. These 2 cases made us aware of this complication as a potential pitfall and allowed us to take suitable steps to prevent it in the future. The vertebral artery follows an oblique course from its origin at the first part of the subclavian artery. It runs along the su- 
TABLE 3. Complications with $\mathrm{cC}-7$ nerve dissection and transection

\begin{tabular}{lccl}
\hline \multicolumn{1}{c}{ Complication } & No. of Patients & Outcome \\
\hline Mistook varied C-6 for C-7 & 1 & No apparently changed motor function \\
\hline Transection of posterior division of the lower trunk & 1 & $\begin{array}{c}\text { Decreased elbow \& wrist extension; recovered, but } \\
\text { finger \& thumb extensor strength is Grade 0 }\end{array}$ \\
\hline Posterior division of the lower trunk, partial injury & 3 & All recovered \\
\hline Pain in healthy extremity & 3 & All recovered \\
\hline Horner's syndrome & 2 & $\begin{array}{c}1 \text { fully recovered \& } 1 \mathrm{w} / \text { no ptosis or miosis but still } \\
\text { had anhidrosis }\end{array}$ \\
\hline Atrophy of sternocostal part of the pectoralis major muscle & 1 & No recovery but no functional change \\
\hline
\end{tabular}

perior part of the longus colli muscle and then deep into it. It lies anterior to the intervertebral foramen between the C-6 and C-7 and then enters the lateral mass of the C-7 transverse process. ${ }^{1}$ The C-7 nerve emerges at this level. Preparation of the passage from the intervertebral foramen to the retroesophageal space requires us to traverse deep to the scalenus anterior muscle and through the longus colli muscle. Blind insertion of a blunt hemostat carries the risk of injury to the vertebral vessels. Bleeding from the vertebral vein becomes evident as oozing along the tube passed to transfer the nerve to the injured side. It can be controlled by local pressure and bipolar coagulation. A rent in the vertebral artery produces heavy bleeding that is difficult to control. Recently, we modified our technique to prevent this complication (shown in Figs. 6-8). The fibers of the longus colli muscle are carefully separated to expose the vertebral vessels. If they lie within the superficial portion of the muscle and can be isolated easily, the nerve

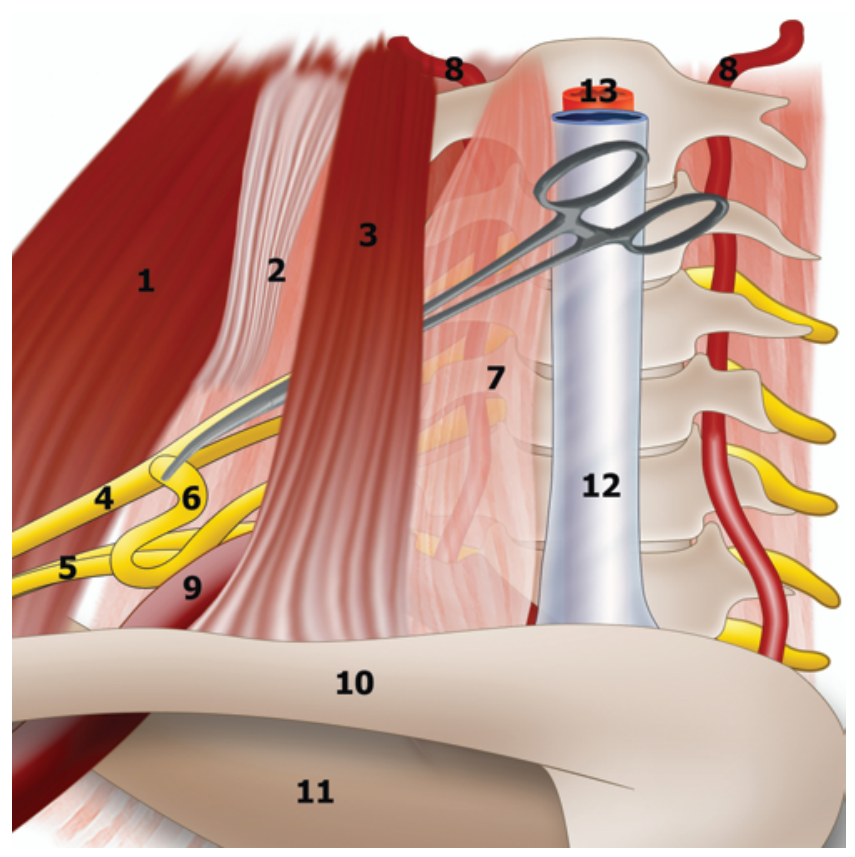

FIG. 6. Schematic of the modified $\mathrm{CC}-7$ nerve root passed under the anterior scalene muscle. $1=$ trapezius; $2=$ middle scalene muscle; $3=$ anterior scalene muscle; $4=$ upper trunk; $5=$ lower trunk; $6=$ cC-7; 7 $=$ longus colli; 8 = vertebral artery; $9=$ subclavian artery; 10 = clavicle; 11 = first rib; 12 = trachea; 13 = esophagus. Copyright Xiaoping Li. Published with permission. Figure is available in color online only. is passed deep to the vessels along the anterolateral aspect of the vertebral body. Conversely, if the vessels cannot be separated easily or are lying deep within the muscle, the nerve is passed superficially to the vertebral vessels. In any case, the nerve is transferred after visualization of the vessels at risk.

One of our patients died in the perioperative period. He had undergone a splenectomy and had suffered multiple injuries, including cervical vertebral body fractures, cervical spondylolisthesis, and several fractures in his extremities. He had normal function in the opposite upper limb before the operation. However, his reflexes were brisk and his general condition was poor. The period immediately after surgery was uneventful. However, the patient developed dyspnea 42 hours postoperatively. A clinical physical examination revealed a partial palsy of the opposite

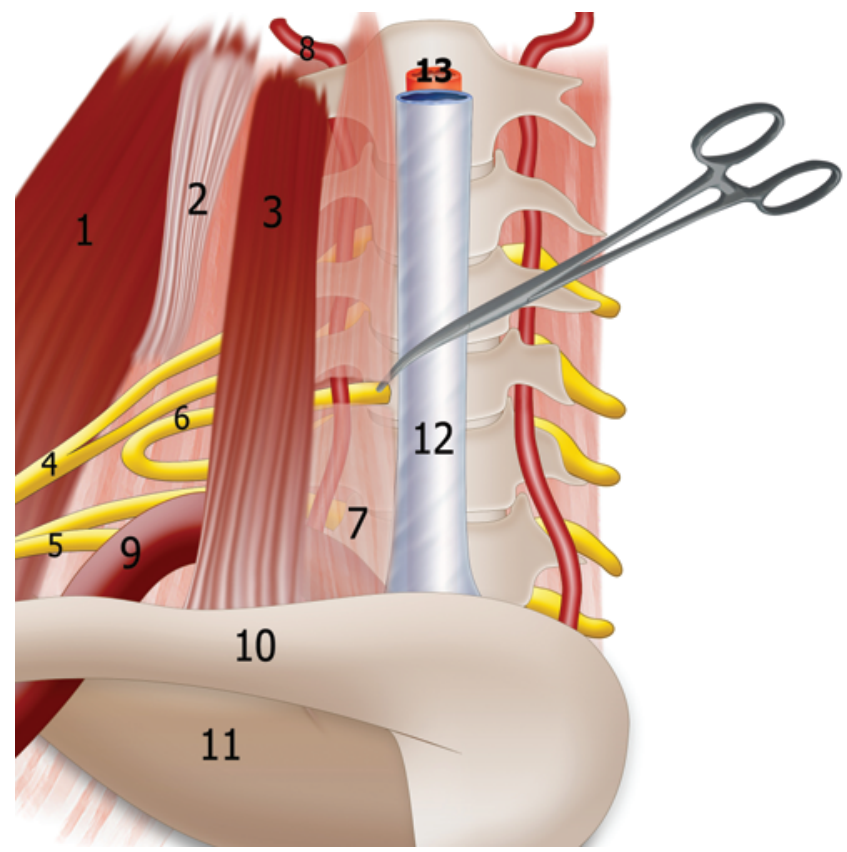

FIG. 7. Schematic of the modified procedure for protection of the vertebral artery from laceration. We carefully separated and sectioned the longus colli to expose the vertebral vessels and then passed the cC-7 nerve deep or superficial to the vessels. $1=$ trapezius; $2=$ middle scalene muscle; $3=$ anterior scalene muscle; $4=$ upper trunk; $5=$ lower trunk; 6 = cC-7; 7 = longus colli; $8=$ vertebral artery; $9=$ subclavian artery; 10 = clavicle; 11 = first rib; 12 = trachea; 13 = esophagus. Copyright Xiaoping Li. Published with permission. Figure is available in color online only. 


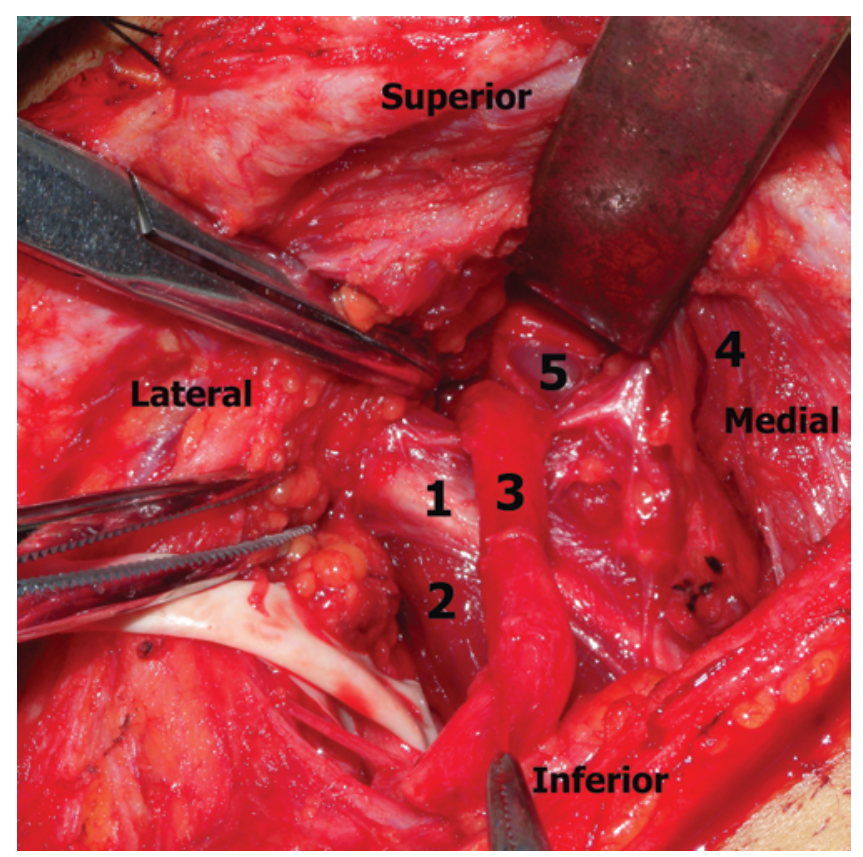

FIG. 8. Under direct vision of the vertebral artery and vein when the cC-7 nerve root was transferred through the prevertebral route. $1=$ phrenic nerve; 2 = anterior scalene muscle; $3=\mathrm{cC}-7$ nerve root, which passed under the anterior scalene muscle; $4=$ transected longus colli; 5 $=$ vertebral artery and vein. Superior, inferior, lateral, and medial are the orientations. Figure is available in color online only.

upper limb and weakness in the lower extremities and in the muscles of the tongue. The pathological reflexes were positive in both lower limbs. The patient was fully conscious throughout this period. The preliminary diagnosis was thrombosis with ischemia of the brainstem. A tracheostomy was performed, and the patient was transferred to the intensive care unit. He eventually died as a result of cardiac and respiratory failure 38 days after the operation. It is not certain whether this complication was attributable to the prespinal route. However, it made us more prudent in selecting patients for this procedure.

\section{Complications Arising From Dissection and Transection of cC-7 Root}

We were especially interested to note any complications that may have occurred as a result of cC-7 nerve transection. Our fears were based on the potential for anatomical variations found during exploration and the possibility of upper-limb weakness on the noninjured side after the patient recovered from anesthesia. In our cases, we cut the cC-7 root after separating it until it reached the distal end of its division. In this way, we could get the longest possible nerve. To procure a greater length for transfer across the midline, this inevitably brings us closer to the intersections with the upper and lower trunks. A mere sectioning of the C-7 nerve root would not cause any perceptible deficit. However, damage to the adjacent trunks must be avoided at all costs. In our series, there were 4 patients who suffered weakness on extension of the fingers and of the thumb. In 1 of these patients, the deficit was caused by the accidental severance of the posterior division of the inferior trunk. In the other 3 , weakness in the territory of the posterior division of the inferior trunk was attributed to repeated electrical stimulation and handling for electrophysiology examination. The posterior cord, formed from the confluence of the posterior divisions of the upper, middle, and lower trunks, continues into the radial nerve. The posterior divisions of the middle and inferior trunks supply extension of the fingers and of the thumb. ${ }^{9}$ When the C-7 root is divided completely, function depends on innervation from the posterior division of the lower trunk. Hence, one must avoid injury to this structure while harvesting the C-7 root. As the C-7 root is traced distally, we visualize the converging posterior divisions of the upper and lower trunks. The posterior division of the lower trunk lies in a deeper plane to the respective segment of the middle trunk, and accidental traction injury should be avoided. Crossing fibers from the posterior division of the lower trunk are carefully protected. In addition, the lower trunk must be examined before transection of the posterior division of the C-7 nerve. In 1 of these 4 patients, there was no recovery of thumb or finger extension (Fig. 4) because the posterior division of the lower trunk was not repaired. In the other 3 cases, the deficit resolved gradually because the posterior division of the lower trunk had been preserved in continuity.

Mistakenly sectioning other nerves instead of the C-7 nerve for transfer can cause severe dysfunction of the contralateral upper arm, which would be a catastrophe for the patient. This complication has not yet been reported. Careful dissection of the entire plexus would help in the proper identification of the C-7 nerve so that such a complication can be avoided. This is particularly true when the C-7 root splits early into its divisions or in obese patients with short necks.

The 3 patients who had pain in their contralateral arm recovered fully. This complication may have been caused by injury to a few fibers of the superior trunk when the divisions of the $\mathrm{cC}-7$ root were being separated distally.

The appearance of Horner's sign on the healthy side in 2 of our patients with obstetrical palsies is difficult to explain. In a rat model, Huang et al. ${ }^{8}$ tried to elucidate the relationship between sympathetic preganglionic fibers and the brachial plexus. They found that, in young rats, some of the sympathetic preganglionic fibers pass along the C-7 root to the superior cervical ganglion. This pathway disappears during the postnatal period. Thus, the Horner sign seen in obstetrical palsies may correspond to avulsion of the C-7 root (and not the C-8 and T-1 roots as described for adult palsies). Hence, the appearance of this sign in our cases may be attributed to accidental injury to the tiny branches carrying the sympathetic fibers close to the intervertebral foramen. No other complications occurred in our obstetrical palsy cases.

\section{Other Complications}

We did not note any cases of infection, lymphedema, or lymphatic leakage.

\section{Conclusions}

The most serious complications seen in our series were damage to the vertebral vessels and lesions of the poste- 
rior division of the lower trunk. If a vascular injury occurs, we recommend immediate repair by microvascular techniques or ligation. Injury to the posterior division of the lower trunk can be avoided by careful dissection while harvesting the $\mathrm{C}-7$ nerve root.

The complication rate in our large series of patients was low, and even the types of complications that did occur can be avoided. Hence, we feel that the superior results obtained by direct repair justify the widespread use of this technique in the future.

\section{Acknowledgment}

We thank Anil G. Bhatia, M.D. (Department of Hand Surgery, Joshi Hospital, Fortis Hospital, Maharashtra, India), for his editorial assistance.

\section{References}

1. Alterman DM, Heidel RE, Daley BJ, Grandas OH, Stevens SL, Goldman MH, et al: Contemporary outcomes of vertebral artery injury. J Vasc Surg 57:741-746, 2013

2. Chuang DC, Hernon C: Minimum 4-year follow-up on contralateral C7 nerve transfers for brachial plexus injuries. J Hand Surg Am 37:270-276, 2012

3. Feng J, Wang T, Gu Y, Chen L, Zhang G, Zhu Y: Contralateral C7 transfer to lower trunk via a subcutaneous tunnel across the anterior surface of chest and neck for total root avulsion of the brachial plexus: a preliminary report. Neurosurgery 66 (6 Suppl Operative):252-263, 2010

4. Gu Y, Xu J, Chen L, Wang H, Hu S: Long term outcome of contralateral C7 transfer: a report of 32 cases. Chin Med J (Engl) 115:866-868, 2002

5. Gu YD, Cai PQ, Xu F, Peng F, Chen L: Clinical application of ipsilateral C7 nerve root transfer for treatment of C5 and C6 avulsion of brachial plexus. Microsurgery 23:105-108, 2003

6. Gu YD, Chen DS, Zhang GM, Cheng XM, Xu JG, Zhang LY, et al: Long-term functional results of contralateral C7 transfer. J Reconstr Microsurg 14:57-59, 1998

7. Gu YD, Zhang GM, Chen DS, Yan JG, Cheng XM, Chen L: Seventh cervical nerve root transfer from the contralateral healthy side for treatment of brachial plexus root avulsion. J Hand Surg Br 17:518-521, 1992

8. Huang YG, Chen L, Gu YD, Yu GR: Sympathetic preganglionic neurons project to superior cervical ganglion via $\mathrm{C} 7$ spinal nerve in pup but not in adult rats. Auton Neurosci 154:54-58, 2010

9. Li WJ, Wang SF, Li PC, Li YC, Jin YD, Yang Y, et al: Electrophysiological study of the dominant motor innervation to the extensor digitorum communis muscle and long head of triceps brachii at posterior divisions of brachial plexus. Microsurgery 31:535-538, 2011

10. McGuiness CN, Kay SP: The prespinal route in contralateral C7 nerve root transfer for brachial plexus avulsion injuries. J Hand Surg Br 27:159-160, 2002

11. Wang S, Yiu HW, Li P, Li Y, Wang H, Pan Y: Contralateral C7 nerve root transfer to neurotize the upper trunk via a modified prespinal route in repair of brachial plexus avulsion injury. Microsurgery 32:183-188, 2012

12. Wang SF, Li PC, Xue YH, Yiu HW, Li YC, Wang HH: Contralateral C7 nerve transfer with direct coaptation to restore lower trunk function after traumatic brachial plexus avulsion. J Bone Joint Surg Am 95:821-827, S1-S2, 2013

13. Xu L, Gu Y, Xu J, Lin S, Chen L, Lu J: Contralateral C7 transfer via the prespinal and retropharyngeal route to repair brachial plexus root avulsion: a preliminary report. Neurosurgery 63:553-559, 2008

\section{Author Contributions}

Conception and design: Wang. Acquisition of data: Wang, W $\mathrm{Li}, \mathrm{P} \mathrm{Li}$, Xue. Analysis and interpretation of data: W Li, Zhao, Y Li, P Li. Drafting the article: W Li. Critically revising the article: all authors. Reviewed submitted version of manuscript: all authors. Approved the final version of the manuscript on behalf of all authors: Wang. Statistical analysis: Zhao, Y Li, P Li, Xue. Administrative/technical/material support: W Li, Xue. Study supervision: Wang, Zhao, Y Li, P Li, Xue.

\section{Correspondence}

Shufeng Wang, Xin Jie Kou Dong Jie 31hao, Xichengqu, Beijing 100035, China. email: wangshufeng1964@aliyun.com. 